\title{
Progressive stenosis and radiological findings of vasculitis over the entire internal carotid artery in moyamoya vasculopathy associated with graves' disease: a case report and review of the literature
}

Hiroto Ito ${ }^{1^{*}}$, Syunsuke Yokoi ${ }^{2}$, Kinya Yokoyama ${ }^{3}$, Takumi Asai ${ }^{3}, K^{2}$ Keni Uda ${ }^{4}$, Yoshio Araki ${ }^{4}$, Syuntaro Takasu ${ }^{5}$, Rei Kobayashi ${ }^{1}$, Hisashi Okada ${ }^{1}$ and Satoshi Okuda ${ }^{1}$

\begin{abstract}
Background: Moyamoya vasculopathy (MMV) associated with Graves' disease (GD) is a rare condition resulting in ischemic stroke accompanied by thyrotoxicity. Radiological findings of vasculitis have been reported in the walls of distal internal carotid arteries (ICAs) in these patients; however, no reports have described in detail the processes of progression of the lesions in the proximal ICA. Moreover, treatments to prevent recurrence of ischemic stroke and progression of MMV have not yet been sufficiently elucidated.

Case presentation: We report a progressive case of MMV associated with GD and review the literature to clarify relationships among recurrence, progression, thyrotoxicity and treatment. Our patient developed cerebral infarction during thyrotoxicity with no obvious stenosis of ICAs. Five months later, transient ischemic attacks recurred with thyrotoxicity. Antiplatelet therapy and intravenous methylprednisolone stopped the attacks. Stenosis of the left ICA from the proximal to distal portion and champagne bottle neck sign (CBN) were found. She declined any surgery. Afterward, gradual progression with mild thyrotoxicity was observed. Eventually, we found smooth, circumferential, concentric wall thickening with diffuse gadolinium enhancement of the left ICA from the proximal to the distal portion on T1-weighted imaging, suggesting vasculitis radiologically. The clinical and radiological similarities to Takayasu arteritis encouraged us to provide treatment as for vasculitis of medium-to-large vessels. In a euthyroid state and after administration of prednisolone and methotrexate, improved flow in the cerebrovascular arteries on magnetic resonance angiography was observed. Based on our review of the literature, all cases with recurrence or progression were treated with anti-thyroid medication (ATM) alone and accompanied by thyrotoxicity. CBN was observed in all previous cases for which images of the proximal ICA were available.

(Continued on next page)
\end{abstract}

\footnotetext{
* Correspondence: hiroto.ito.nagoya.uni.med@gmail.com

${ }^{1}$ Department of Neurology, National Hospital Organization Nagoya Medical

Center, 4-1-1 Sannomaru, Naka-ku, Nagoya, Aichi, Japan

Full list of author information is available at the end of the article
}

C The Author(s). 2019 Open Access This article is distributed under the terms of the Creative Commons Attribution 4.0 International License (http://creativecommons.org/licenses/by/4.0/), which permits unrestricted use, distribution, and reproduction in any medium, provided you give appropriate credit to the original author(s) and the source, provide a link to the Creative Commons license, and indicate if changes were made. The Creative Commons Public Domain Dedication waiver (http://creativecommons.org/publicdomain/zero/1.0/) applies to the data made available in this article, unless otherwise stated. 
(Continued from previous page)

Conclusions: We report the details of progressive stenosis from a very early stage and radiological findings of vasculitis over the entire ICA in MMV associated with GD. Cerebral infarction can occur with no obvious stenosis of the ICA. We treated the patient as per vasculitis of a medium-to-large vessel. Management of GD by ATM alone seems risky in terms of recurrence. Adequate management of GD and possible vasculitis may be important for preventing recurrence and progression.

Keywords: Moyamoya vasculopathy, Graves' disease, Recurrence, Champagne bottle neck sign, Vasculitis

\section{Background}

Graves' disease (GD) is rarely complicated by moyamoya vasculopathy (MMV), resulting in ischemic stroke during thyrotoxicity $[1,2]$. Management of GD is considered important to prevent recurrence [3], but MMV may progress despite the control of GD [4]. A case was recently reported in which the wall of the distal ICA was enhanced on contrast-enhanced (CE) T1-weighted imaging (T1WI), suggesting vasculitis [5]. However, no reports have described the details of the processes of progression and the lesions of the proximal ICA in MMV associated with GD. Moreover, results have not been described in these patients after treatment for vasculitis of medium-to-large vessels and several issues remain uncertain, such as treatments to prevent recurrence and progression, and characteristic radiological findings.

\section{Case presentation}

A 37-year-old woman presented with gradually progressing weakness of the right arm. She had a medical history of asthma only in her childhood and no notable family history. On physical examination, she showed mild paralysis of the right arm. Although she felt palpitation and sweating at times, exophthalmic and enlarged thyroid lobes were not observed. Diffusion-weighted imaging (DWI) showed cortical and subcortical infarcts in the left MCA territory (Additional file 1: Figure S1), but magnetic resonance angiography (MRA) showed almost-normal cerebral arteries or very mild stenosis of the left ICA (Figs. 1a, 2a). The vessel wall seemed thicker in the left ICA than in the right on three-dimensional (3D)-T1WI, but the difference was not clear (Additional file 2: Figure S2A). Hyperthyroidism [levels of free T3, free T4, and thyroid stimulating hormone (TSH); $10.58 \mathrm{pg} / \mathrm{mL}, 2.70$ $\mathrm{ng} / \mathrm{dL}$, and $0.01 \mu \mathrm{U} / \mathrm{mL}$, respectively], and autoantibodies related to GD [anti-thyroid peroxidase antibody (anti-TPO $\mathrm{Ab}, 148.0 \mathrm{IU} / \mathrm{mL}$ ), and TSH receptor antibody (TRAb, 8.3 $\mathrm{IU} / \mathrm{mL}$ )] were identified (Fig. 3). Other laboratory investigations showed unremarkable results except for leukopenia, anti-SS-A antibody $(89.5 \mathrm{IU} / \mathrm{mL}$; normal range, < $7.0 \mathrm{IU} /$ $\mathrm{mL})$, anti-SS-B antibody $(12.4 \mathrm{IU} / \mathrm{mL}$; normal range, $<7.0$ $\mathrm{IU} / \mathrm{mL}$ ) and thrombin-antithrombin complex (TAT, $2.5 \mathrm{ng} /$ $\mathrm{mL}$; normal range, $<0.3 \mathrm{ng} / \mathrm{mL})$. C-reactive protein $(\mathrm{CRP})$ and erythrocyte sedimentation rate (ESR) were negative. She was diagnosed with GD, but not with Sjogren's syndrome, based on the normal results of Schirmer's test and a salivary flow-rate test. Rather than methimazole, she was treated with potassium iodide $(150 \mathrm{mg} /$ day) for GD due to leukopenia. MRA excludes over 50\% stenosis of intracranial and extracranial cerebral arteries and we could not find any major risks of cardioembolic source of embolism, through electrocardiography, echocardiography, and cardiac rhythm monitoring for over $24 \mathrm{~h}$, so heparin, then warfarin $(4 \mathrm{mg} /$ day) were administrated as treatments for stroke as unknown etiology. Mild weakness remained at discharge.

Five months later, she presented with intermittent transient weakness of the right arm and leg. DWI did not show new infarction. Stenosis of the left ICA progressed on MRA (Figs. 1b, 2b). Thyrotoxicity was exacerbated (Fig. 3). Although argatroban was administered, attacks recurred 5 times. We started intravenous methylprednisolone (IVMP; $1000 \mathrm{mg} /$ day on days 2-6), clopidogrel (300 mg on day 1 , and $75 \mathrm{mg} /$ day from day 2), and aspirin (300 $\mathrm{mg}$ on day 1 , and $100 \mathrm{mg}$ /day from day 2 ). The attacks subsequently stopped. Catheter angiography showed stenosis of the left ICA from the proximal to distal portion and CBN (Fig. 4a, b). High intensity lesion on T1 W1 in the distal portion of the left ICA was observed (Additional file 2: Fig. S2B). MMV associated with GD was diagnosed. She refused any surgery. Methimazole (15 mg/day) was started, but mild thyrotoxicity continued (Fig. 3).

We subsequently identified gradual progression in the left ICA and middle cerebral artery (MCA) on MRA (Fig. 1b-d) and 3D computed tomographic angiography. Finally, we found smooth, circumferential, concentric wall thickening with diffuse gadolinium enhancement of the left ICA from the proximal to distal portion on 3D-T1WI (Fig. 5a, b). We considered the possibility of vasculitis of medium-to-large vessels and administered prednisolone (PSL; $1 \mathrm{mg} / \mathrm{kg} /$ day) and methotrexate (MTX; $4 \mathrm{mg} /$ week). Subsequently, thyroid function normalized (Fig. 3).

Six months later, PSL was reduced to $0.1 \mathrm{mg} / \mathrm{kg} /$ day, and MTX increased to $14 \mathrm{mg} /$ week. Signal intensity in the left ICA and MCA increased on MRA. Catheter angiography showed development of net-like vessels, 


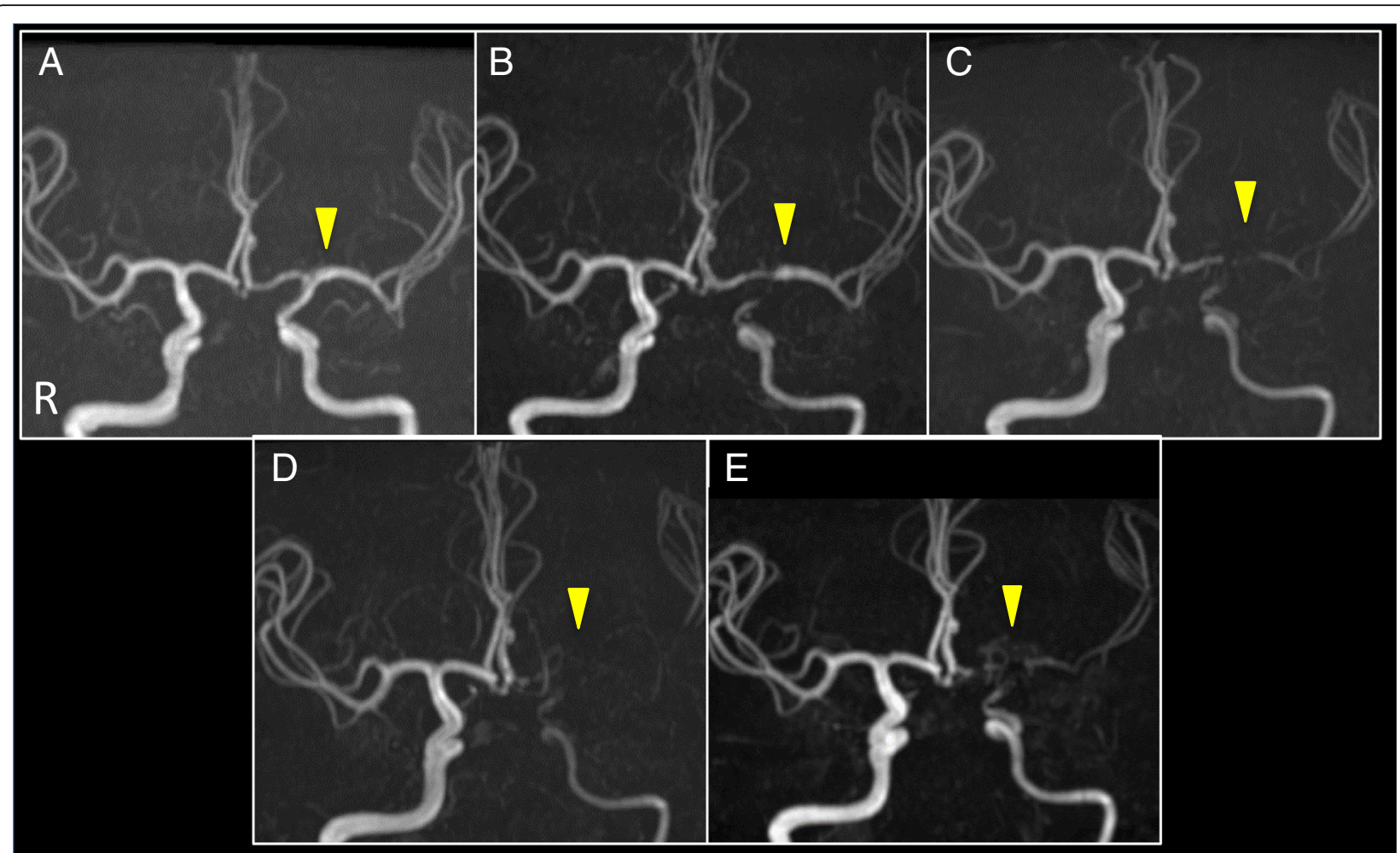

Fig. 1 Brain magnetic resonance angiography in the course. a Brain MRA did not show obvious stenosis of ICAs. b-d Stenosis in cerebral arteries on brain MRA progressed during thyrotoxicity. e Eighteen months after recurrence, brain MRA suggests improved blood flow through improved flow of the left ICA and MCA

and incomplete occlusion of the ICA (Fig. 4c). By 18 months after recurrence, further improved flow in the left ICA and MCA on MRA was observed (Fig. 1e), but the vessel wall of the left ICA from the proximal to the distal portion still remained enhanced on CE 3D-T1WI in the same way as the previous one. Two years after recurrence, catheter angiography also showed improved blood flow in the left ACA, MCA and ICA, as well as mild improvement of stenosis of the terminal portion of the left ICA (Fig. 4d). We evaluated the polymorphism in c. $14576 \mathrm{G}>\mathrm{A}$ (rs112735431) in the RNF-213 gene, a susceptibility gene for Moyamoya disease, using genomic DNA samples from the patient. Genetic analysis of RNF-213 showed wild type.

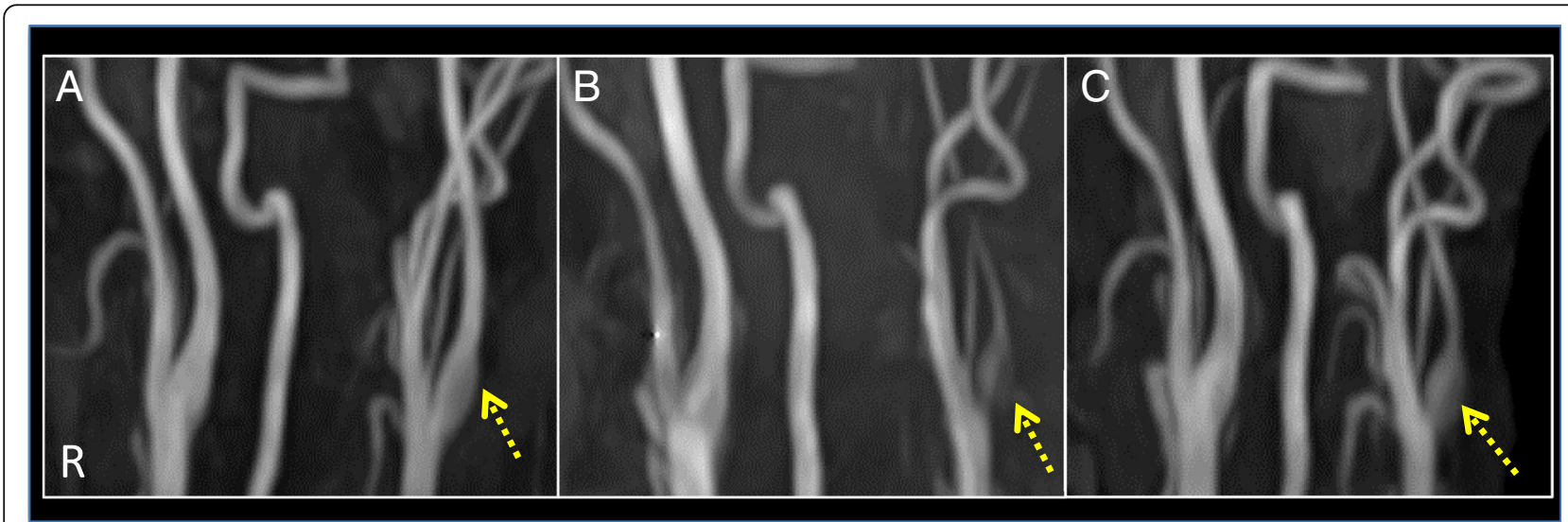

Fig. 2 Cervical magnetic resonance angiography in the course. a Cervical MRA showed very mild or no obvious stenosis in the first episode. $\mathbf{b}$ Cervical MRA showed progressed stenosis of the left ICA at proximal portion and CBN was observed in the second episode for the first time. $\mathbf{c}$ Eighteen months after recurrence, cervical MRA also suggests improved blood flow through improved flow in the proximal left ICA 


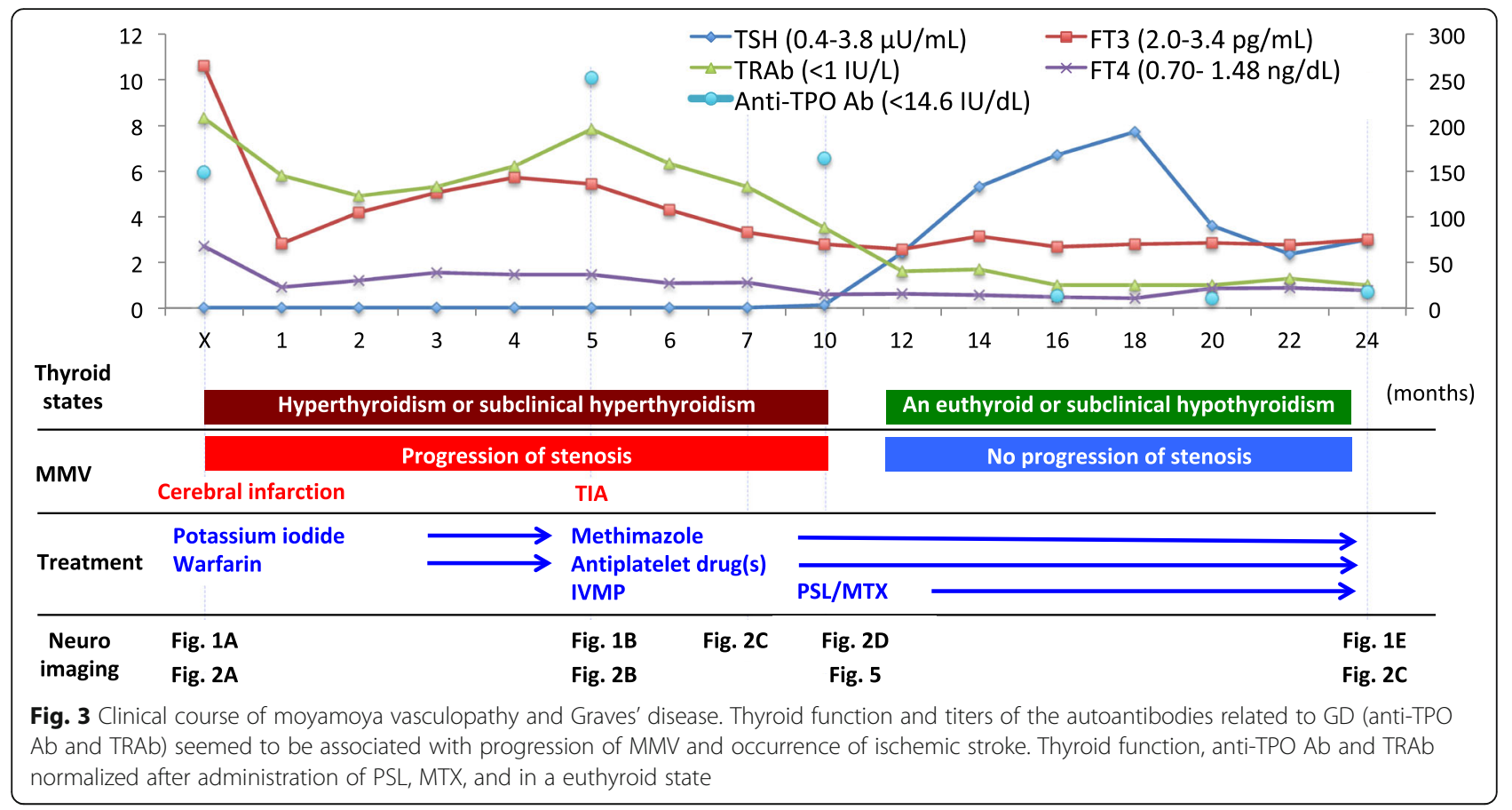

\section{Discussion and conclusions}

\section{Review of the literature}

We reported a patient with recurrent ischemic stroke and progressive stenosis of MMV associated with GD who had no obvious stenosis in the first stroke. We also reviewed previous reports with follow-up for over 4 months to clarify long-term relationships among recurrence of ischemic stroke, progression of MMV, thyrotoxicity and treatment. We conducted a Medline search for articles using the key words "cerebrovascular diseases", "moyamoya", "thyrotoxicity", and "Graves' disease". To confirm long-term relationships, we selected case reports followed-up for over 4 months among these candidates [1, 2, 6-18], and excluded review articles and cases showing recurrence or repeated transient ischemic attacks within 3 months (Table 1), because we wanted to know whether treatment and control of GD over the long term was associated with recurrent ischemic stroke or progression of MMV. We also examined the presence of stenosis of ICAs, and champagne bottle neck sign $(\mathrm{CBN})$ in all candidates [19].

\section{Patients}

The 20 patients included, 19 female and 1 male, with a mean age of 30.1 years (range, 15-54 years). The majority of the patients were female. Mean duration of follow-up was 22.3 months (range, 4-72 months).

\section{Thyrotoxicity and cerebrovascular disease in the first episodes}

Eighteen patients (90\%) showed neurologic symptoms during thyrotoxicity. One patient $(5 \%)$ presented with subclinical thyrotoxicity and one patient (5\%) was in a hypothyroid state.

\section{Treatment}

For GD, 14 patients $(70 \%)$ were treated with anti-thyroid medication (ATM), and 10 of these patients (50\%) with ATM alone. Two patients (10\%) were treated with only thyroidectomy, 2 patients (10\%) with PSL and radioactive iodine therapy (RIT), and 1 patient (5\%) with PSL and thyroidectomy. For MMV, 8 patients (40\%) were treated with antiplatelet therapy, 1 patient (5\%) with anticoagulant, and 4 patients $(20 \%)$ with neurovascular surgery. There was no report of patients with no treatment.

\section{Outcomes-Recurrence and progression}

Six patients (30\%) experienced recurrence, and 5 patients (25\%) showed progression. All of them showed thyrotoxic states at recurrence or progression and were treated with ATM alone for GD. One patient died of recurrence [17]. In contrast, among 5 patients (25\%) treated with thyroidectomy or RIT, no patient experienced recurrence or progression. Three patients (15\%) in a euthyroid state showed improved flow in the cerebrovascular arteries on MRA.

\section{Radiographic features}

Based on our investigation of previous reports in the literature, our case represents the first description of ischemic stroke with no obvious stenosis of the cerebral arteries. Images of the proximaI ICA were available in 7 cases $[1,5,12,14,20-22]$. CBN was found in all cases. 


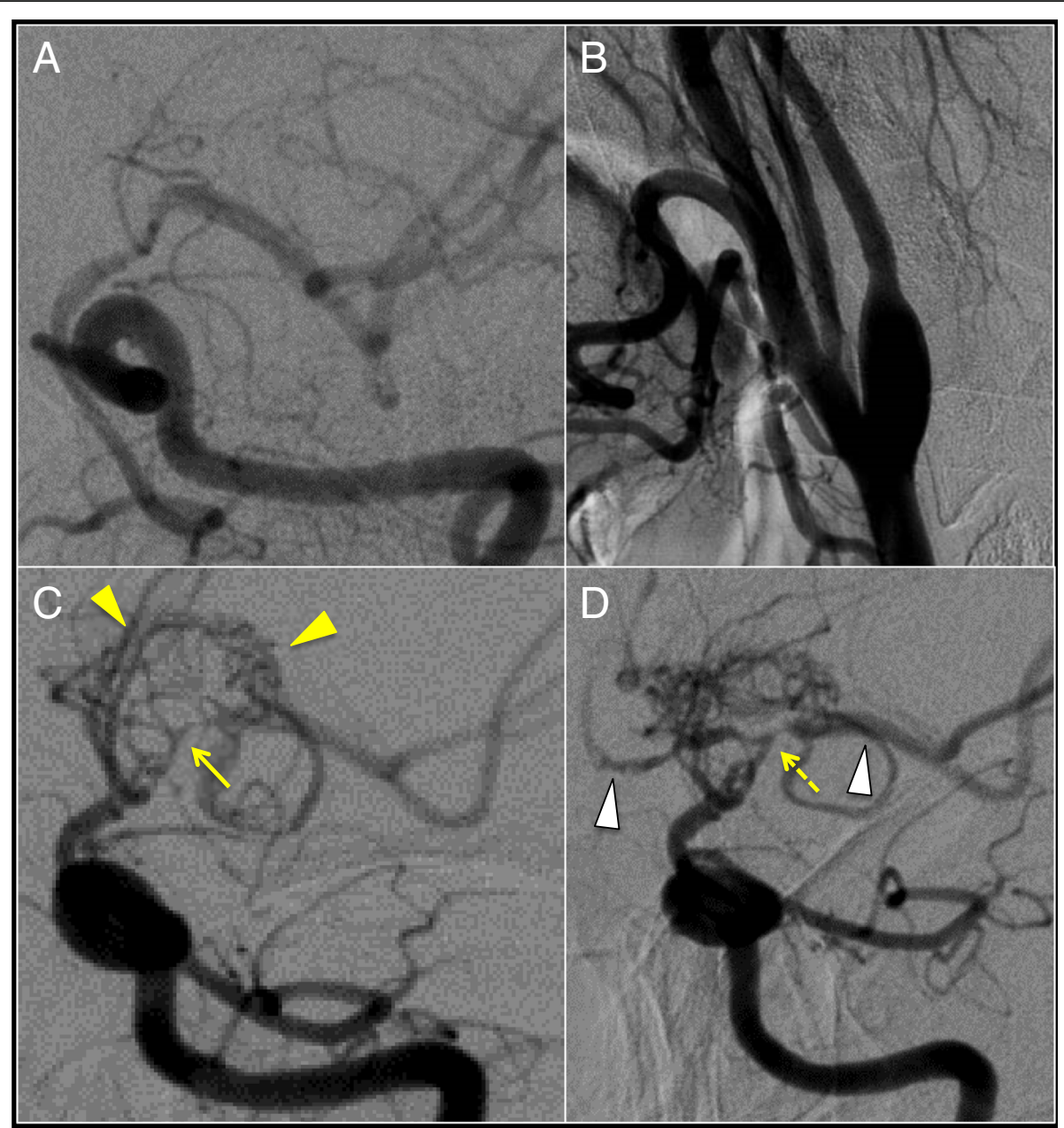

Fig. 4 Cerebral angiograms in the second episode, 1 year and 2 years later. a Cerebral angiograms of the terminal portion of the left ICA in the second episode showed severe stenosis of the distal portion. $\mathbf{b}$ Cerebral angiograms of the proximal left ICA showed stenosis at proximal ICA and CBN in the second episode. c Cerebral angiograms 12 months after the second episode showed progression of stenosis of the distal portion and net-like vessels might have been developed (arrowhead-yellow), but incomplete occlusion (arrow). d Cerebral angiograms 24 months after the second episode showed improved blood flow in the left ACA, MCA and ICA (arrowhead-white), as well as mild improvement of stenosis of the terminal portion of the left ICA (dashed arrow). We consider that since the progression of MMV stopped after administration of PSL and MTX, and in the euthyroid state, cerebral angiograms did not show complete occlusion of distal ICA. Afterward, the net-like vessels might have developed only in the limited space around the distal ICA region. Improved blood flow through the net-like vessels and mild improvement of stenosis in cerebral arteries on cerebral angiograms might have increased blood flow in the left ICA and MCA on MRA

The clinical course of our case is illustrated in Fig. 1. Thyroid function and titers of anti-TPO Ab and TRAb seemed to be associated with progression of MMV and occurrence of ischemic stroke. We found smooth, circumferential, concentric wall thickening with diffuse gadolinium enhancement over the entire left ICA, which is the radiological finding of vasculitis [23, 24]. Improved flow in the left ICA and MCA on MRA was observed after administration of PSL and MTX, and in the euthyroid state. The result of genetic analysis of RNF-213 also suggests a different etiology of Moyamoya disease associated with c. $14576 \mathrm{G}>\mathrm{A}$ variant in the RNF-213 gene.

We noticed similarities between our case and Takayasu arteritis, including the involvement of a young female, findings of medium-to-large vessels on CE MR imaging, progression of stenosis in medium-to-large vessels, and elevated TAT $[25,26]$. CRP and ESR can be negative in Takayasu arteritis [26]. In the first episode of our case, a hemodynamic etiology appeared unlikely as the cause of ischemic stroke, because stenosis of the left ICA was not obvious. It has been reported that although little, if any, endothelial change is evident, thrombus formation by vasculitis leads to ischemic stroke in Takayasu arteritis [25]. Thus, the same mechanism can be considered in our case. In the second episode, high intensity lesion on T1 W1 in the distal portion of the left ICA was observed (Additional file 2: Figure S2B). Dissection might be considered as the etiology of this lesion, because dissection shows eccentric 


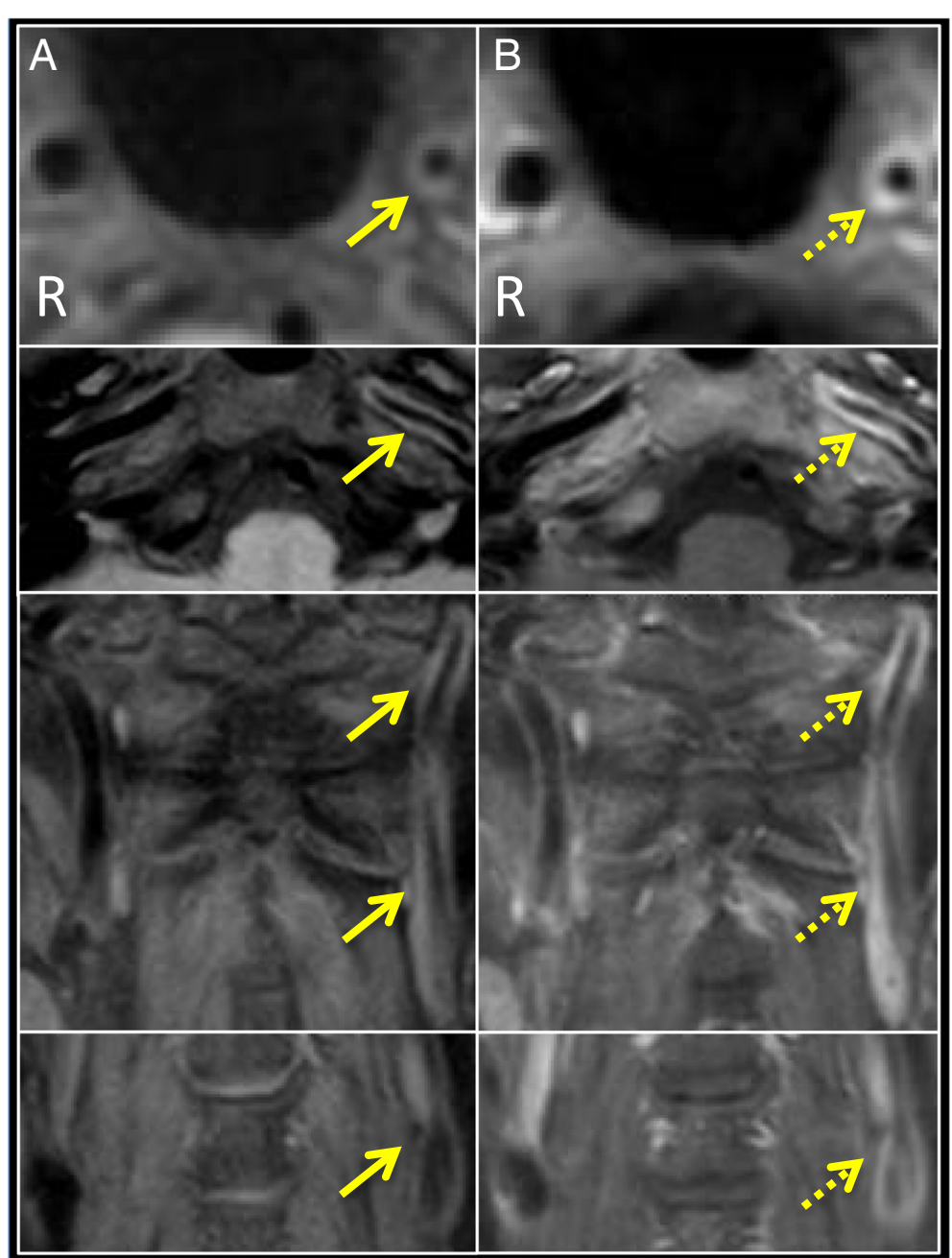

Fig. 5 Radiological findings of vasculitis over the entire internal carotid artery. Six months after recurrence, 3D-T1WI (a) and CE 3D-T1WI (b) of the ICAs were performed (Upper 2 figures: axial images of the distal portion; Lower 2 figures: coronal images of the proximal portion in Figure $\mathbf{a}$, $\mathbf{b}$ ). $\mathbf{a}$ 3D-T1WI showed smooth, concentric wall thickening over the entire left ICA (arrow). b CE 3D-T1WI showed diffuse contrast enhancement on vessel walls (dashed arrow in Figure b) over the entire left ICA, suggesting vasculitis radiologically [23, 24]

wall thickening with $\mathrm{T} 1$ bright wall components representing intramural hematoma [24]. Moreover, vasculitis can cause aortic dissection, for example, Takayasu arteritis. T-cell-mediated immunity was reported to play important roles in Takayasu arteritis, GD and MMV [26-28]. Immunologic changes related to GD and MMV may have a common pathogenic link involving T-cell dysregulation [28]. The association between MMV and GD in our case and our review of the literature support this hypothesis.

CBN may be a characteristic feature of this disease and may be caused by vasculitis from the proximal ICA. CBN means a rapid, sharp reduction in internal diameter at the proximal ICA, which is observed in some patients with moyamoya disease [6]. In our case, the difference between thickness of the wall of the left and right ICA seemed to become clear gradually (Fig. 5a, b Additional file 2: Figure S2A, B), possibly due to vasculitis. This seemed to contribute to the formation of stenosis of the entire ICA and CBN in our case (Additional file 3: Figure S3). In other cases of MMV associated with GD or moyamoya disease with $\mathrm{CBN}$, the same process might occur. Since the vessel lumen is narrower in the distal than in the proximal portion, occlusion may occur in the distal portion.

Treatment for MMV associated with GD by ATM alone may be risky in terms of recurrence. So far, the treatments, especially without surgery, have not been sufficiently studied. For GD, ATM, RIT, thyroidectomy, PSL and PE were used in previous reports. For MMV, treatments should reportedly be performed the same way as for moyamoya disease through antiplatelet agents and bypass surgery [29]. The similarities with Takayasu arteritis encouraged us to treat our patient with PSL and MTX as treatments for vasculitis, although, to the best 


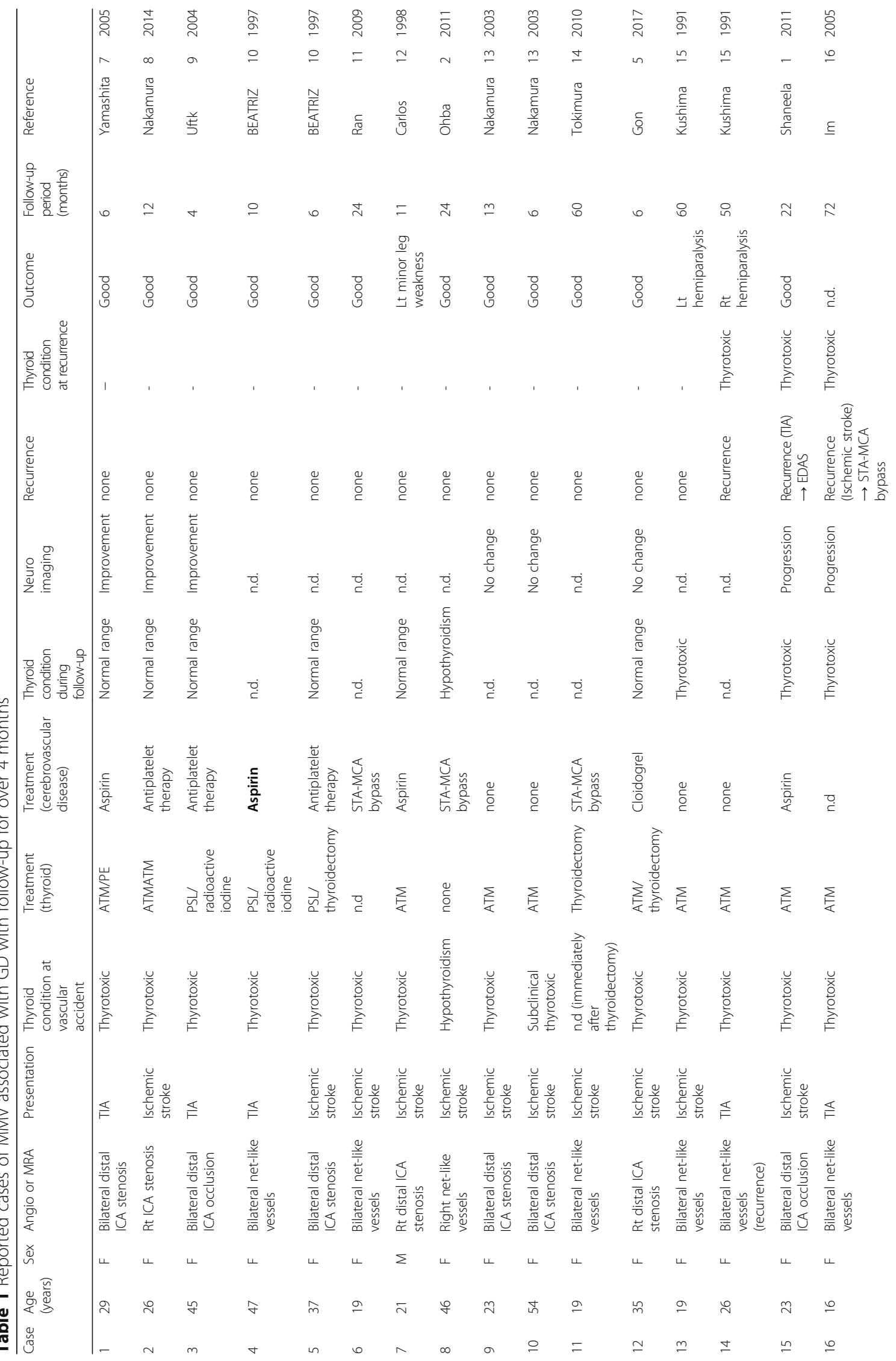




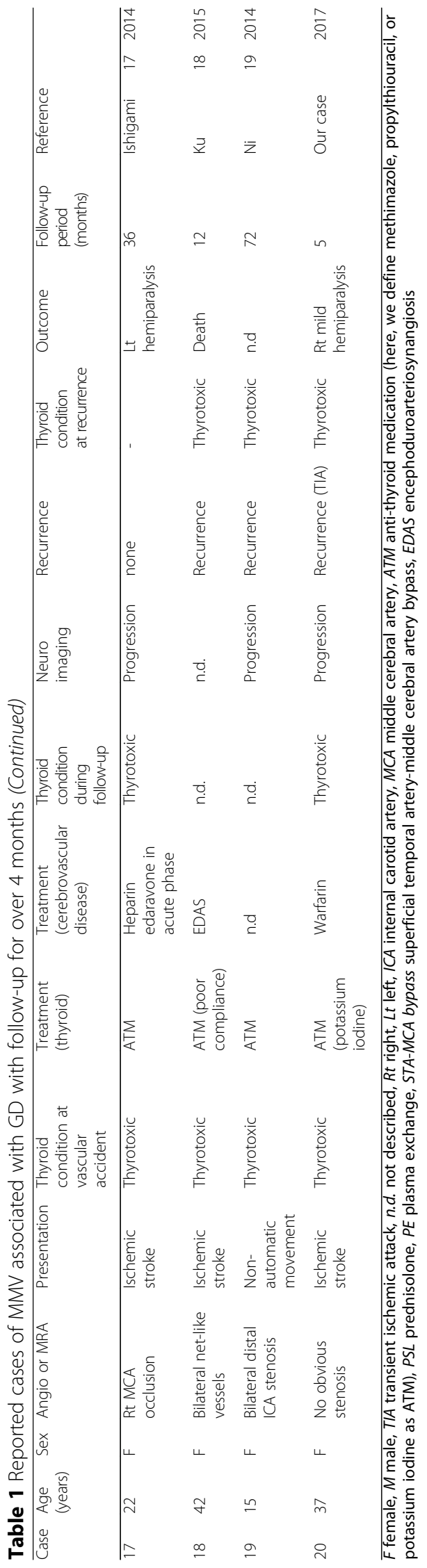


of our knowledge, there has been no report of such treatment for this type of patient. Improved flow of the left ICA and MCA on MRA was observed after administration of PSL and MTX, and in the euthyroid state. On the other hand, such a favorable result, the improved flow, can not occur in Moyamoya disease, in which MCA disappears as the next stage in the Suzuki stage [29]. Considering a retrospective study in which progression advanced even when GD was controlled [4], the improvement on MRA in our case might have been due to the treatment of vasculitis. IVMP in the acute phase in the second episode might have worked for both thyrotoxicity and vasculitis. According to our review, recurrence or progression was found in patients treated with ATM alone, while recurrence or progression was not found with RIT or thyroidectomy. This seems to be due to the higher recurrence rate (range, 50-67\%) for GD under the usual treatment with ATM compared to $15 \%$ with RIT and $10 \%$ with thyroidectomy [30]. Notably, 1 patient treated with ATM alone died following recurrence [17]. Since RIT was reported to increase the risk of cerebrovascular events [31], thyroidectomy may be reasonable choice to reduce the risk of recurrence or probable progression.

Our review was limited by the retrospective nature of the data collection. For example, duration of follow-up tended to be longer in recurrent cases. Despite these limitations, the overall review provides important lessons on recurrence.

\section{Conclusions}

This report suggests the possibility of vasculitis of medium-to-large vessels in MMV associated with GD. This is the first case where cerebral infarction occurred during thyrotoxicity without obvious stenosis of ICAs. We treated the patient as having vasculitis of medium-to-large vessels with IVMP in the acute phase and with PSL and MTX in the chronic phase. Subsequently, improved flow in the cerebrovascular arteries on MRA was observed in the euthyroid state. Treatment with ATM alone seems to be risky in terms of recurrence. Adequate management of GD and possible vasculitis may be important for preventing recurrence and progression.

\section{Additional files}

Additional file 1: Figure S1. MR imaging of cerebral infarction in the first episode. Axial diffusion-weighted images showed infarcts in the left cerebral hemisphere. (PDF $202 \mathrm{~kb}$ )

Additional file 2: Figure S2. Coronal 3D-T1WI of ICA in the first and the second episode. (A): Image in the first episode. The vessel walls might be thicker over the left entire ICA compared to the right, but it was not clear. (PDF $322 \mathrm{~kb}$ ) (B): Image in the second episode. Stenosis became severe, especially in the distal portion of the left ICA. High intensity lesion on T1 W1 in the distal portion of the left ICA (arrow) was observed.
Dissection might be considered as the etiology of this lesion, because dissection shows eccentric wall thickening with $\mathrm{T} 1$ bright wall components representing intramural hematoma [24]. Moreover, vasculitis can cause aortic dissection, for example, Takayasu arteritis.

Additional file 3: Figure S3. Hypothetical process for the formation of the CBN and stenosis over the entire ICA and its relationship to MMV. We hypothesize that vessel wall thickness by vasculitis contributes to the formation of the CBN and stenosis of the entire left ICA on MRA. This is because they became more evident in the second episode than in the first episode in our case. Since the vessel lumen is narrower in the distal than in the proximal portion, occlusion may occur in the distal portion. (PDF $204 \mathrm{~kb}$ )

\section{Abbreviations}

3D: three-dimensional; anti-TPO Ab: anti-thyroid peroxidase antibody; ATM: anti-thyroid medication; CBN: champagne bottle neck sign; CE: contrast-enhanced; CRP: C-reactive protein; DWI: diffusion-weighted imaging; ESR: erythrocyte sedimentation rate; GD: Graves' disease; ICA: internal carotid artery; IVMP: intravenous methylprednisolone; MCA: middle cerebral artery; MMV: moyamoya vasculopathy; MRA: magnetic resonance angiography; MTX: methotrexate; PSL: prednisolone; RIT: radioactive iodine therapy; T1WI: T1-weighted imaging; TAT: thrombinantithrombin complex; TRAb: TSH receptor antibody; TSH: thyroid stimulating hormone

\section{Acknowledgements}

The authors are grateful to Dr. Yasufumi Gon (Osaka University), Dr. Masaaki Ito (Nagoya Univaersity), Dr. Kazunori Shintai, Dr. Hideki lijima (Nagoya Daini Redcross Hospital), and Kiichi Miyamae (Nagoya Medical Center) for useful discussion and advice.

Funding

None of the authors have received any financial assistance for this work.

Availability of data and materials

All data generated or analyzed during this study are included in this published article.

\section{Authors' contributions}

$\mathrm{HI}, \mathrm{SY}, \mathrm{KY}, \mathrm{TA}, \mathrm{RK}, \mathrm{HO}$, SO made the clinical diagnosis, and clinical management decisions. They supervised the drafting of the manuscript. HI drafted the first manuscript and reviewed the literature. RK and SO revised it critically. KU, YA and ST supported making the diagnosis and clinical management decisions as the specialists of Moyamoya disease and performed genetic analysis of RNF-213. They also supervised the drafting of the manuscript and revised it critically. All authors have read and approved the final manuscript.

Ethics approval and consent to participate

Not applicable.

\section{Consent for publication}

Written informed consent was obtained from the patient for publication of this case report. A copy of the written consent is available for review by the Editor of this journal.

Competing interests

The authors declare that they have no competing interests.

\section{Publisher's Note}

Springer Nature remains neutral with regard to jurisdictional claims in published maps and institutional affiliations.

\section{Author details}

'Department of Neurology, National Hospital Organization Nagoya Medical Center, 4-1-1 Sannomaru, Naka-ku, Nagoya, Aichi, Japan. ²Department of Rheumatology, National Hospital Organization Nagoya Medical Center, 4-1-1 Sannomaru, Naka-ku, Nagoya, Aichi, Japan. ${ }^{3}$ Department of Neurosurgery, National Hospital Organization Nagoya Medical Center, 4-1-1 Sannomaru, 
Naka-ku, Nagoya, Aichi, Japan. ${ }^{4}$ Department of Neurosurgery, Nagoya University Graduate School of Medicine, Tsurumai-cho 65, Showa-ku, Nagoya, Aichi, Japan. ${ }^{5}$ Department of Neurosurgery, Japanese Red Cross Nagoya Daini Hospital, 2-9 Myouken-cho, Showa-ku, Nagoya, Aichi, Japan.

\section{Received: 29 August 2018 Accepted: 19 February 2019}

\section{Published online: 02 March 2019}

\section{References}

1. Malik S, Russman AN, Katramados AM, Silver B, Mitsias PD. Moyamoya syndrome associated with graves' disease: a case report and review of the literature. J Stroke Cerebrovasc Dis. 2011;(6):528-36.

2. Ohba S, Nakagawa T, Murakami H. Concurrent graves' disease and intracranial arterial stenosis/occlusion: special considerations regarding the state of thyroid function, etiology, and treatment. Neurosurg Rev. 2011;34: 297-304.

3. Shah NH, Khandelwal P, Gordon-Perue G, Shah AH, Barbarite E, Ortiz G, et al. Acute thyrotoxicosis of graves disease associated with moyamoya vasculopathy and stroke in Latin American women: a case series and review of the literature. World Neurosurg. 2016;92:95-107.

4. Chen JB, Lei D, He M, Sun H, Liu Y, Zhang H, et al. Clinical features and disease progression in moyamoya disease patients with graves disease. $J$ Neurosurg. 2015;123:848-55.

5. Gon Y, Sakaguchi M, Oyama N, Mochizuki H. Diagnostic utility of contrastenhanced 3D T1-weighted imaging in acute cerebral infarction associated with graves disease. J Stroke Cerebrovasc Dis. 2017;26(2):38-40.

6. Yamashita S, Tamiya T, Shindo A, Miyake K, Nakamura T, Ogawa D, et al. Improvement of cerebral arterial stenosis associated with Basedow's disease. Case report Neurol Med Chir (Tokyo). 2005;45:578-82.

7. Nakamura H, Kosuge $Y$, Mizuniwa $Y$, Wakui D, Taguchi $Y$. A case of reversible stenosis in the cervical internal carotid artery causing cerebral infarction associated with Basedow disease. Jpn J Stroke. 2014;36:51-3.

8. Tendler BE, Shoukri K, Malchoff C, MacGillivray D, Duckrow R, Talmadge T, et al. Concurrence of graves' disease and dysplastic cerebral blood vessels of the moyamoya variety. Thyroid. 1997;7:625-9.

9. Utku U, Asil T, Celik Y, Tucer D. Reversible MR angiographic findings in a patient with autoimmune graves disease. AJNR Am J Neuroradiol. 2004; 25(9):1541-3.

10. Lee R, Sung K, Park YM, Yu JJ, Koh YC, Chung S. A case of Moyamoya disease in a girl with thyrotoxicosis. Yonsei Med J. 2009;50:594-8.

11. Leno C, Mateo I, Cid C, Berciano J, Sedano C. Autoimmunity in Down's syndrome: another possible mechanism of moyamoya disease. Stroke. 1998; 29:868-9.

12. Nakamura K, Yanaka K, Ihara S, Nose T. Multiple intracranial arterial stenosis around the circle of Willis in association with graves' disease: report of two cases. Neurosurgery. 2003:53:1210-4.

13. Tokimura H, Tajitsu K, Takashima H, Hirayama T, Tsuchiya M, Takayama K, et al. Familial Moyamoya disease associated with Graves' disease in a mother and daughter. Neurol Med Chir (Tokyo). 2010;50:668-74.

14. Kushima K, Satoh Y, Ban Y, Taniyama M, Ito K, Sugita K. Graves' thyrotoxicosis and moyamoya disease. Can J Neurol Sci. 1991;18:140-2.

15. Im SH, Oh CW, Kwon OK, Kim JE, Han DH. Moyamoya disease associated with graves disease: special considerations regarding clinical significance and management. J Neurosurg. 2005:102:1013-7.

16. Ishigami A, Toyoda K, Suzuki R, Miyashita F, lihara K, Minematsu K. Neurologic improvement without angiographic improvement after antithyroid therapy in a patient with Moyamoya syndrome. J Stroke Cerebrovasc Dis. 2014;23(5):1256-8.

17. Ku BD, Park KC, Yoon SS. Fatal ischemic stroke in a case of progressive moyamoya vasculopathy associated with uncontrolled thyrotoxicosis. Korean J Intern Med. 2015;30(4):543-6.

18. Ni J, Zhou LX, Wei YP, Li ML, Xu WH, Gao S, et al. Moyamoya syndrome associated with Graves' disease: a case series study. Ann Transl Med. 2014; 2(8):77.

19. Yasaka M, Ogata T, Yasumori $K$, Inoue T, Okada Y. Bottle neck sign of the proximal portion of the neck appearance in moyamoya disease. $J$ Ultrasound Med. 2006;25(12):1547-52.

20. Liu JS, Juo SH, Chen WH, Chang YY. Chen SS. A case of graves' diseases associated with intracranial moyamoya vessels and tubular stenosis of extracranial internal carotid arteries. J Formos Med Assoc. 1994;93(9):806-9.
21. Shen AL, Ryu SJ, Lin SK. Concurrent moyamoya disease and graves' thyrotoxicosis: case report and literature review. Acta Neurol Taiwanica. 2006;15:114-9.

22. Shimogawa T, Morioka T, Sayama T, Hamamura T, Yasuda C, Arakawa S. Champagne bottle neck sign in a patient with Moyamoya syndrome. World J Clin Cases. 2014;2(9):474-7.

23. Choi YJ, Jung SC, Lee DH. Vessel wall imaging of the intracranial and cervical carotid arteries. J Stroke. 2015;17:238-55.

24. Swartz RH, Bhuta SS, Farb RI, Agid R, Willinsky RA, terBrugge KG, et al. Intracranial arterial wall imaging using high-resolution 3-tesla contrastenhanced MRI. Neurology. 2009:72:627-34.

25. Akazawa H, Ikeda U, Yamamoto K, Kuroda T, Shimada K. Hypercoagulable state in patients with Takayasu's arteritis. Thromb Haemost. 1996:75(5):712-6.

26. Keser G, Direskeneli H, Aksu K. Management of Takayasu arteritis: a systematic review. Rheumatology (Oxford). 2014:53(5):793-801.

27. Soliman M, Kaplan E, Yanagawa T, Hidaka Y, Fisfalen ME, DeGroot LJ. T-cells recognize multiple epitopes in the human thyrotropin receptor extracellular domain. J Clin Endocrinol Metab. 1995;80(3):905-14.

28. Panegyres PK, Morris JG, O'Neill PJ, Balleine R. Moyamoya-like disease with inflammation. Eur Neurol. 1993:33:260-3.

29. Fujimura M, Tominaga T. Diagnosis of moyamoya disease: international standard and regional differences. Neurol Med Chir (Tokyo). 2015:55:189-93.

30. Brent GA. Graves' disease. N Engl J Med. 2008;358(24):2594-605.

31. la Cour JL, Jensen LT, Vej-Hansen A, Nygaard B. Radioiodine therapy increases the risk of cerebrovascular events in hyperthyroid and euthyroid patients. Eur J Endocrinol. 2015;172(6):771-8.

\section{Ready to submit your research? Choose BMC and benefit from:}

- fast, convenient online submission

- thorough peer review by experienced researchers in your field

- rapid publication on acceptance

- support for research data, including large and complex data types

- gold Open Access which fosters wider collaboration and increased citations

- maximum visibility for your research: over $100 \mathrm{M}$ website views per year

At $\mathrm{BMC}$, research is always in progress.

Learn more biomedcentral.com/submissions 